HU e-ISSN 1787-2413

\title{
NOTES ON EXPLICIT AND INVERSION FORMULAS FOR THE CHEBYSHEV POLYNOMIALS OF THE FIRST TWO KINDS
}

\author{
FENG QI, DA-WEI NIU, AND DONGKYU LIM
}

Received 27 May, 2019

\begin{abstract}
In the paper, starting from the Rodrigues formulas for the Chebyshev polynomials of the first and second kinds, by virtue of the Faà di Bruno formula, with the help of two identities for the Bell polynomials of the second kind, and making use of a new inversion theorem for combinatorial coefficients, the authors derive two nice explicit formulas and their corresponding inversion formulas for the Chebyshev polynomials of the first and second kinds.
\end{abstract}

2010 Mathematics Subject Classification: 11B38; 11C08; 26C05; 33C45; 33C47; 33D45

Keywords: explicit formula, inversion formula, Rodrigues formula, Chebyshev polynomial of the first kind, Chebyshev polynomial of the second kind, Faà di Bruno formula, Bell polynomial of the second kind

\section{INTRODUCTION}

It is well known $[5-7,31]$ that the Chebyshev polynomials of the first and second kinds $T_{n}$ and $U_{n}(x)$ are very important in mathematical sciences and that, in the study of ordinary differential equations [5, pp. xxxv and 1004], they arise as solutions to the Chebyshev differential equations

$$
\left(1-x^{2}\right) y^{\prime \prime}-x y^{\prime}+n^{2} y=0 \text { and }\left(1-x^{2}\right) y^{\prime \prime}-3 x y^{\prime}+n(n+2) y=0
$$

for the Chebyshev polynomials of the first and second kinds $T_{n}$ and $U_{n}$ respectively.

In [6, Eqs. (4.30) and (4.31)], the Rodrigues formulas for the Chebyshev polynomials of the first and second kinds $T_{n}$ and $U_{n}$ read that

$$
T_{n}(x)=(-1)^{n} \frac{2^{n} n !}{(2 n) !}\left(1-x^{2}\right)^{1 / 2} \frac{\mathrm{d}^{n}}{\mathrm{~d} x^{n}}\left[\left(1-x^{2}\right)^{n-1 / 2}\right]
$$

and

$$
U_{n}(x)=(-1)^{n} \frac{2^{n}(n+1) !}{(2 n+1) !}\left(1-x^{2}\right)^{-1 / 2} \frac{\mathrm{d}^{n}}{\mathrm{~d} x^{n}}\left[\left(1-x^{2}\right)^{n+1 / 2}\right] .
$$

For variants of the Rodrigues formulas for the Chebyshev polynomials of the first and second kinds $T_{n}$ and $U_{n}$, please refer to, for example, [5, pp. 1003-1004], [7, p. 442], [19, Section 4], and [31, pp. 432-433]. 
In [5, p. 1003], the Rodrigues formulas for $T_{n}(x)$ and $U_{n}(x)$ are written in the forms

$$
T_{n}(x)=(-1)^{n} \frac{\sqrt{1-x^{2}}}{(2 n-1) ! !} \frac{\mathrm{d}^{n}}{\mathrm{~d} x^{n}}\left[\left(1-x^{2}\right)^{n-1 / 2}\right]
$$

and

$$
U_{n}(x)=\frac{(-1)^{n}(n+1)}{\sqrt{1-x^{2}}(2 n+1) ! !} \frac{\mathrm{d}^{n}}{\mathrm{~d} x^{n}}\left[\left(1-x^{2}\right)^{n+1 / 2}\right] .
$$

In [5, p. 1004] and [31, pp. 432-433], the Rodrigues formulas for $T_{n}(x)$ and $U_{n}(x)$ are formulated as

$$
T_{n}(x)=\frac{(-1)^{n} \sqrt{\pi}}{2^{n} \Gamma(n+1 / 2)}\left(1-x^{2}\right)^{1 / 2} \frac{\mathrm{d}^{n}}{\mathrm{~d} x^{n}}\left[\left(1-x^{2}\right)^{n-1 / 2}\right]
$$

and

$$
U_{n}(x)=\frac{(-1)^{n} \sqrt{\pi}(n+1)}{2^{n+1} \Gamma(n+3 / 2)}\left(1-x^{2}\right)^{-1 / 2} \frac{\mathrm{d}^{n}}{\mathrm{~d} x^{n}}\left[\left(1-x^{2}\right)^{n+1 / 2}\right],
$$

where $\Gamma(z)$ stands for the classical gamma function which can be defined $[8,16]$ by

$$
\Gamma(z)=\lim _{n \rightarrow \infty} \frac{n ! n^{z}}{\prod_{k=0}^{n}(z+k)}, \quad z \in \mathbb{C} \backslash\{0,-1,-2, \ldots\}
$$

or by

$$
\Gamma(z)=\int_{0}^{\infty} t^{z-1} e^{-t} \mathrm{~d} t, \quad \Re(z)>0 .
$$

In [7, p. 442], the Rodrigues formulas for $T_{n}(x)$ and $U_{n}(x)$ are arranged as

$$
T_{n}(x)=\frac{\left(1-x^{2}\right)^{1 / 2}}{(-2)^{n}(1 / 2)_{n}} \frac{\mathrm{d}^{n}}{\mathrm{~d} x^{n}}\left[\left(1-x^{2}\right)^{n-1 / 2}\right]
$$

and

$$
U_{n}(x)=\frac{(n+1)\left(1-x^{2}\right)^{-1 / 2}}{(-2)^{n}(3 / 2)_{n}} \frac{\mathrm{d}^{n}}{\mathrm{~d} x^{n}}\left[\left(1-x^{2}\right)^{n+1 / 2}\right],
$$

where $(x)_{n}$ for $n \geq 0$ and $x \in \mathbb{R}$ denotes the rising factorial which can be defined [22] by

$$
(x)_{n}=\prod_{\ell=0}^{n-1}(x+\ell)=\frac{\Gamma(x+n)}{\Gamma(x)}= \begin{cases}x(x+1) \cdots(x+n-1), & n \geq 1 \\ 1, & n=0 .\end{cases}
$$

By virtue of the recurrence relation $\Gamma(x+1)=x \Gamma(x)$, we have

$$
\Gamma\left(n+\frac{1}{2}\right)=\prod_{\ell=0}^{n-1}\left(n-\ell-\frac{1}{2}\right) \Gamma\left(\frac{1}{2}\right)=\left(\frac{1}{2}\right)_{n} \sqrt{\pi}=\frac{(2 n-1) ! !}{2^{n}} \sqrt{\pi}
$$


and

$$
\Gamma\left(n+\frac{3}{2}\right)=\prod_{\ell=0}^{n}\left(n-\ell+\frac{1}{2}\right) \Gamma\left(\frac{1}{2}\right)=\left(\frac{3}{2}\right)_{n} \frac{\sqrt{\pi}}{2}=\frac{(2 n+1) ! !}{2^{n+1}} \sqrt{\pi} .
$$

Substituting these into (1.5) and (1.6) respectively leads to (1.3), (1.4), (1.7), and (1.8) which are equivalent to (1.1) and (1.2) respectively.

In [31, pp. 432-433], it was listed that

$$
T_{n}(x)=\frac{n}{2} \sum_{m=0}^{\lfloor n / 2\rfloor}(-1)^{m} \frac{(n-m-1) !}{m !(n-2 m) !}(2 x)^{n-2 m}
$$

and

$$
U_{n}(x)=\sum_{m=0}^{\lfloor n / 2\rfloor}(-1)^{m} \frac{(n-m) !}{m !(n-2 m) !}(2 x)^{n-2 m},
$$

where $n \in \mathbb{N}$ and $\lfloor t\rfloor$ denotes the floor function whose value equals the largest integer less than or equal to $t$.

In this paper, starting from the four formulas (1.1), (1.2), (1.9), and (1.10), by virtue of the Faà di Bruno formula, with the help of two identities for the Bell polynomials of the second kind, and making use of a new inversion theorem [28, Theorem 4.3] for combinatorial coefficients, we will derive the following two nice explicit formulas and their corresponding inversion formulas for the Chebyshev polynomials $T_{n}$ and $U_{n}$.

\section{FOUR LEMMAS}

For proving our main results, Theorems 1 and 2 below, we need the following four lemmas.

Lemma 1 ([4, pp. 134 and 139]). For $n \geq k \geq 0$, the Faà di Bruno formula can be described in terms of the Bell polynomials of the second kind $\mathrm{B}_{n, k}\left(x_{1}, x_{2}, \ldots, x_{n-k+1}\right)$ by

$$
\frac{\mathrm{d}^{n}}{\mathrm{~d} t^{n}} f \circ h(t)=\sum_{k=0}^{n} f^{(k)}(h(t)) \mathrm{B}_{n, k}\left(h^{\prime}(t), h^{\prime \prime}(t), \ldots, h^{(n-k+1)}(t)\right) .
$$

Lemma 2 ([4, p. 135]). For $n \geq k \geq 0$, we have

$$
\mathrm{B}_{n, k}\left(a b x_{1}, a b^{2} x_{2}, \ldots, a b^{n-k+1} x_{n-k+1}\right)=a^{k} b^{n} \mathrm{~B}_{n, k}\left(x_{1}, x_{2}, \ldots, x_{n-k+1}\right),
$$

where $a$ and $b$ are any complex numbers. 
Lemma 3 ([13, Theorem 4.1] and [27, Section 3]). For $n \geq k \geq 0$, the Bell polynomials of the second kind $\mathrm{B}_{n, k}\left(x_{1}, x_{2}, \ldots, x_{n-k+1}\right)$ satisfy

$$
\mathrm{B}_{n, k}(x, 1,0, \ldots, 0)=\frac{1}{2^{n-k}} \frac{n !}{k !}\left(\begin{array}{c}
k \\
n-k
\end{array}\right) x^{2 k-n},
$$

where $\left(\begin{array}{l}0 \\ 0\end{array}\right)=1$ and $\left(\begin{array}{l}p \\ q\end{array}\right)=0$ for $q>p \geq 0$.

Lemma 4 ([28, Theorem 4.3]). For $n \geq k \geq 1$, let $s_{k}$ and $S_{k}$ be two sequences independent of $n$. Then

if and only if

$$
\frac{s_{n}}{n !}=\sum_{k=1}^{n}(-1)^{k}\left(\begin{array}{c}
k \\
n-k
\end{array}\right) S_{k}
$$

$$
n S_{n}=\sum_{k=1}^{n} \frac{(-1)^{k}}{(k-1) !}\left(\begin{array}{c}
2 n-k-1 \\
n-1
\end{array}\right) s_{k} .
$$

\section{MAIN RESUltS AND THEIR PROOFS}

Now we begin to state and prove our main results, Theorems 1 and 2 below.

Theorem 1. For $n \geq 0$, the Chebyshev polynomials $T_{n}$ and $U_{n}$ can be explicitly computed by

$$
T_{n}(x)=x^{n} \sum_{\ell=0}^{\lfloor n / 2\rfloor}\left(\begin{array}{c}
n \\
2 \ell
\end{array}\right)\left(1-\frac{1}{x^{2}}\right)^{\ell}
$$

and

$$
U_{n}(x)=x^{n} \sum_{\ell=0}^{\lfloor n / 2\rfloor}\left(\begin{array}{c}
n+1 \\
2 \ell+1
\end{array}\right)\left(1-\frac{1}{x^{2}}\right)^{\ell} .
$$

Proof. By virtue of the formuals (2.1), (2.2), and (2.3), we have

$$
\begin{gathered}
\frac{\mathrm{d}^{n}}{\mathrm{~d} x^{n}}\left[\left(1-x^{2}\right)^{n-1 / 2}\right]=\sum_{k=1}^{n} \frac{\mathrm{d}^{k} u^{n-1 / 2}}{\mathrm{~d} u^{k}} \mathrm{~B}_{n, k}(-2 x,-2,0 \ldots, 0) \\
=\sum_{k=1}^{n} \prod_{\ell=0}^{k-1}\left(n-\ell-\frac{1}{2}\right) u^{n-k-1 / 2}(-2)^{k} \mathrm{~B}_{n, k}(x, 1,0 \ldots, 0) \\
=\sum_{k=1}^{n} \frac{1}{2^{k}} \prod_{\ell=0}^{k-1}(2 n-2 \ell-1)\left(1-x^{2}\right)^{n-k-1 / 2}(-2)^{k} \frac{1}{2^{n-k}} \frac{n !}{k !}\left(\begin{array}{c}
k \\
n-k
\end{array}\right) x^{2 k-n} \\
=\frac{n !}{(2 x)^{n}}\left(1-x^{2}\right)^{n-1 / 2} \sum_{k=1}^{n}(-1)^{k}\left(\begin{array}{c}
k \\
n-k
\end{array}\right) \frac{(2 n-1) ! !}{[2(n-k)-1] ! !} \frac{2^{k}}{k !}\left(\frac{x^{2}}{1-x^{2}}\right)^{k}
\end{gathered}
$$




$$
=\frac{n !(2 n-1) ! !}{(2 x)^{n}}\left(1-x^{2}\right)^{n-1 / 2} \sum_{k=1}^{n}(-1)^{k}\left(\begin{array}{c}
k \\
n-k
\end{array}\right) \frac{2^{k}}{k ![2(n-k)-1] ! !}\left(\frac{x^{2}}{1-x^{2}}\right)^{k},
$$

where $n \in \mathbb{N}, u=u(x)=1-x^{2}$, and the double factorial of negative odd integers $-2 n-1$ is defined by

$$
(-2 n-1) ! !=\frac{(-1)^{n}}{(2 n-1) ! !}=(-1)^{n} \frac{2^{n} n !}{(2 n) !}, \quad n \geq 0 .
$$

Substituting the above established equality into (1.1) and simplifying lead to

$$
T_{n}(x)=\sum_{k=1}^{n} \frac{(-1)^{n-k}}{4^{n-k}}\left(\begin{array}{c}
k \\
n-k
\end{array}\right)\left(\begin{array}{l}
n \\
k
\end{array}\right) \frac{[2(n-k)] ! !}{[2(n-k)-1] ! !} x^{2 k-n}\left(1-x^{2}\right)^{n-k}
$$

which can be rearranged, by replacing $n-k$ by $\ell$, as

$$
T_{n}(x)=x^{n} \sum_{\ell=0}^{n-1} \frac{(-1)^{\ell}}{4^{\ell}}\left(\begin{array}{c}
n \\
n-\ell
\end{array}\right)\left(\begin{array}{c}
n-\ell \\
\ell
\end{array}\right) \frac{(2 \ell) ! !}{(2 \ell-1) ! !}\left(\frac{1}{x^{2}}-1\right)^{\ell} .
$$

Since

$$
\frac{1}{4^{\ell}}\left(\begin{array}{c}
n \\
n-\ell
\end{array}\right)\left(\begin{array}{c}
n-\ell \\
\ell
\end{array}\right) \frac{(2 \ell) ! !}{(2 \ell-1) ! !}=\left(\begin{array}{c}
n \\
2 \ell
\end{array}\right)
$$

we arrives at the identity (3.1).

Repeating the above process, we can obtain

$$
\begin{gathered}
\frac{\mathrm{d}^{n}}{\mathrm{~d} x^{n}}\left[\left(1-x^{2}\right)^{n+1 / 2}\right]=\sum_{k=1}^{n} \frac{\mathrm{d}^{k} u^{n+1 / 2}}{\mathrm{~d} u^{k}} \mathrm{~B}_{n, k}(-2 x,-2,0 \ldots, 0) \\
=\sum_{k=1}^{n} \prod_{\ell=0}^{k-1}\left(n-\ell+\frac{1}{2}\right) u^{n-k+1 / 2}(-2)^{k} \mathrm{~B}_{n, k}(x, 1,0 \ldots, 0) \\
=\sum_{k=1}^{n} \frac{1}{2^{k}} \prod_{\ell=0}^{k-1}(2 n-2 \ell+1)\left(1-x^{2}\right)^{n-k+1 / 2}(-2)^{k} \frac{1}{2^{n-k}} \frac{n !}{k !}\left(\begin{array}{c}
k \\
n-k
\end{array}\right) x^{2 k-n} \\
=\frac{n !}{(2 x)^{n}}\left(1-x^{2}\right)^{n+1 / 2} \sum_{k=1}^{n}(-1)^{k}\left(\begin{array}{c}
k \\
n-k
\end{array}\right) \frac{(2 n+1) ! !}{[2(n-k)+1] ! !} \frac{2^{k}}{k !}\left(\frac{x^{2}}{1-x^{2}}\right)^{k} \\
=\frac{n !(2 n+1) ! !}{(2 x)^{n}}\left(1-x^{2}\right)^{n+1 / 2} \sum_{k=1}^{n}(-1)^{k}\left(\begin{array}{c}
k \\
n-k
\end{array}\right) \frac{2^{k}}{k ![2(n-k)+1] ! !}\left(\frac{x^{2}}{1-x^{2}}\right)^{k} .
\end{gathered}
$$

Substituting this into (1.2) and simplifying lead to

$$
U_{n}(x)=\sum_{k=1}^{n} \frac{(-1)^{n-k}}{2^{2 n-2 k+1}}\left(\begin{array}{c}
k \\
n-k
\end{array}\right)\left(\begin{array}{c}
n+1 \\
k
\end{array}\right) \frac{[2(n-k+1)] ! !}{[2(n-k)+1] ! !} x^{2 k-n}\left(1-x^{2}\right)^{n-k} .
$$


Replacing $n-k$ by $\ell$ reveals that

$$
U_{n}(x)=\sum_{\ell=0}^{n-1} \frac{(-1)^{\ell}}{2^{2 \ell+1}}\left(\begin{array}{c}
n+1 \\
n-\ell
\end{array}\right)\left(\begin{array}{c}
n-\ell \\
\ell
\end{array}\right) \frac{[2(\ell+1)] ! !}{(2 \ell+1) ! !} x^{n-2 \ell}\left(1-x^{2}\right)^{\ell} .
$$

Due to

$$
\frac{1}{2^{2 \ell+1}}\left(\begin{array}{c}
n+1 \\
n-\ell
\end{array}\right)\left(\begin{array}{c}
n-\ell \\
\ell
\end{array}\right) \frac{[2(\ell+1)] ! !}{(2 \ell+1) ! !}=\left(\begin{array}{c}
n+1 \\
2 \ell+1
\end{array}\right),
$$

we derive (3.2). The proof of Theorem 1 is complete.

Theorem 2. For $n \in \mathbb{N}$, we have

$$
\sum_{k=1}^{n}\left(\begin{array}{c}
2 n-k-1 \\
n-1
\end{array}\right)(2 x)^{k} T_{k}(x)=\frac{1}{2}(2 x)^{2 n}
$$

and

$$
\sum_{k=1}^{n} k\left(\begin{array}{c}
2 n-k-1 \\
n-1
\end{array}\right)(2 x)^{k} U_{k}(x)=n(2 x)^{2 n} .
$$

Proof. We notice that the formulas (1.9) and (1.10) can be rearranged as

$$
T_{n}(x)=\frac{n}{2} \sum_{m=0}^{\lfloor n / 2\rfloor}(-1)^{m}\left(\begin{array}{c}
n-m \\
m
\end{array}\right) \frac{(2 x)^{n-2 m}}{n-m}
$$

and

$$
U_{n}(x)=\sum_{m=0}^{\lfloor n / 2\rfloor}(-1)^{m}\left(\begin{array}{c}
n-m \\
m
\end{array}\right)(2 x)^{n-2 m} .
$$

The inversion theorem in Lemma 4 can be restated as

$$
(-1)^{n} \frac{S_{n}}{n !}=\sum_{\ell=0}^{n-1}(-1)^{\ell}\left(\begin{array}{c}
n-\ell \\
\ell
\end{array}\right) S_{n-\ell}=\sum_{\ell=0}^{\lfloor n / 2\rfloor}(-1)^{\ell}\left(\begin{array}{c}
n-\ell \\
\ell
\end{array}\right) S_{n-\ell}
$$

if and only if

$$
n S_{n}=\sum_{\ell=1}^{n} \frac{(-1)^{\ell}}{(\ell-1) !}\left(\begin{array}{c}
2 n-\ell-1 \\
n-1
\end{array}\right) s_{\ell} .
$$

The formulas (3.5) and (3.6) can be rearranged as

$$
\frac{2}{n}(2 x)^{n} T_{n}(x)=\sum_{\ell=0}^{\lfloor n / 2\rfloor}(-1)^{\ell}\left(\begin{array}{c}
n-\ell \\
\ell
\end{array}\right) \frac{(2 x)^{2(n-\ell)}}{n-\ell}
$$

and

$$
(2 x)^{n} U_{n}(x)=\sum_{\ell=0}^{\lfloor n / 2\rfloor}(-1)^{\ell}\left(\begin{array}{c}
n-\ell \\
\ell
\end{array}\right)(2 x)^{2(n-\ell)} .
$$


Consequently, we obtain

$$
n \frac{(2 x)^{2 n}}{n}=\sum_{k=1}^{n} \frac{(-1)^{k}}{(k-1) !}\left(\begin{array}{c}
2 n-k-1 \\
n-1
\end{array}\right)(-1)^{k} 2(k-1) !(2 x)^{k} T_{k}(x)
$$

and

$$
n(2 x)^{2 n}=\sum_{k=1}^{n} \frac{(-1)^{k}}{(k-1) !}\left(\begin{array}{c}
2 n-k-1 \\
n-1
\end{array}\right)(-1)^{k} k !(2 x)^{k} U_{k}(x)
$$

which can be simplified as (3.3) and (3.4). The proof of Theorem 2 is complete.

\section{REMARKS}

In this section, we will list several remarks to explain more about the formula (2.3), Lemma 4, our main results, and other things.

Remark 1. To the best of our knowledge, the nice formula (2.3) was first concluded in [13] and has been extensively applied in the papers [9-15, 17, 19,21,23-25,27,29, 30] and closely related references therein. The formula (2.3) has been generalized in the papers $[15,17,20]$ and closely related references therein.

Remark 2. To the best of our knowledge, Lemma 4 is a new inversion theorem and has been applied in the paper $[10,18,19,26]$.

Remark 3. Because both the formula (2.3) and Lemma 4 are new, our main results stated in Theorems 1 and 2, or at least their proofs, are also new.

Remark 4. The Chebyshev polynomials are classical, but their study is still very active. As examples, we recommend three newly-published papers [1-3] to readers. Considering the length of this paper, we would not like to detail main results in these three papers and the closely-related references therein.

Remark 5. This paper is a slightly revised version of the preprint [19].

\section{ACKNOWLEDGEMENTS}

The third author was supported by the National Research Foundation of Korea (Grant No. 2018R1D1A1B07041846).

The authors appreciate anonymous referees for their valuable comments on the original version of this paper.

\section{REFERENCES}

[1] C. Cesarano, "Integral representations and new generating functions of Chebyshev polynomials," Hacet. J. Math. Stat., vol. 44, no. 3, pp. 535-546, 2015, doi: 10.15672/HJMS.20154610029.

[2] C. Cesarano, "Multi-dimensional Chebyshev polynomials: a non-conventional approach," Commun. Appl. Ind. Math., vol. 10, no. 1, pp. 1-19, 2019, doi: 10.1515/caim-2019-0008.

[3] C. Cesarano and C. Fornaro, "A note on two-variable Chebyshev polynomials," Georgian Math. J., vol. 24, no. 3, pp. 339-349, 2017, doi: 10.1515/gmj-2016-0034. 
[4] L. Comtet, Advanced combinatorics, enlarged ed. D. Reidel Publishing Co., Dordrecht, 1974, the art of finite and infinite expansions, doi: 10.1007/978-94-010-2196-8.

[5] I. S. Gradshteyn and I. M. Ryzhik, Table of integrals, series, and products, 8th ed. Elsevier/Academic Press, Amsterdam, 2015, translated from the Russian, Translation edited and with a preface by Daniel Zwillinger and Victor Moll, Revised from the seventh edition [MR2360010], doi: 10.1016/B978-0-12-384933-5.00013-8.

[6] J. C. Mason and D. C. Handscomb, Chebyshev polynomials. Chapman \& Hall/CRC, Boca Raton, FL, 2003.

[7] F. W. J. Olver, D. W. Lozier, R. F. Boisvert, and C. W. Clark, Eds., NIST handbook of mathematical functions. U.S. Department of Commerce, National Institute of Standards and Technology, Washington, DC; Cambridge University Press, Cambridge, 2010, with 1 CD-ROM (Windows, Macintosh and UNIX).

[8] F. Qi, "Limit formulas for ratios between derivatives of the gamma and digamma functions at their singularities," Filomat, vol. 27, no. 4, pp. 601-604, 2013, doi: 10.2298/FIL1304601Q.

[9] F. Qi, "Integral representations for multivariate logarithmic polynomials," J. Comput. Appl. Math., vol. 336, pp. 54-62, 2018, doi: 10.1016/j.cam.2017.11.047.

[10] F. Qi, "A simple form for coefficients in a family of ordinary differential equations related to the generating function of the Legendre polynomials," Adv. Appl. Math. Sci., vol. 17, no. 11, pp. 693-700, 2018.

[11] F. Qi and B.-N. Guo, "Some properties of the hermite polynomials and their squares and generating functions," Preprints, p. 14 pages, 2016, doi: 10.20944/preprints201611.0145.v1.

[12] F. Qi and B.-N. Guo, "Explicit and recursive formulas, integral representations, and properties of the large Schröder numbers," Kragujevac J. Math., vol. 41, no. 1, pp. 121-141, 2017, doi: 10.5937/KgJMath1701121F.

[13] F. Qi and B.-N. Guo, "Explicit formulas for special values of the Bell polynomials of the second kind and for the Euler numbers and polynomials," Mediterr. J. Math., vol. 14, no. 3, pp. Art. 140, 14 pages, 2017, doi: 10.1007/s00009-017-0939-1.

[14] F. Qi and B.-N. Guo, "Several explicit and recursive formulas for the generalized Motzkin numbers," Preprints, p. 11 pages, 2017, doi: 10.20944/preprints201703.0200.v1.

[15] F. Qi and B.-N. Guo, "Viewing some ordinary differential equations from the angle of derivative polynomials," Iran. J. Math. Sci. Inform., vol. 15, no. 2, p. in press, 2020, doi: 10.20944/preprints201610.0043.v1.

[16] F. Qi and W.-H. Li, "Integral representations and properties of some functions involving the logarithmic function," Filomat, vol. 30, no. 7, pp. 1659-1674, 2016, doi: 10.2298/FIL1607659Q.

[17] F. Qi, D.-W. Niu, and B.-N. Guo, "Explicit formulas and identities on Bell polynomials and falling factorials," ResearchGate Preprint, p. 10 pages, 2018, doi: 10.13140/RG.2.2.34679.52640.

[18] F. Qi, D.-W. Niu, and B.-N. Guo, "Some identities for a sequence of unnamed polynomials connected with the Bell polynomials," Rev. R. Acad. Cienc. Exactas Fís. Nat. Ser. A Mat. RACSAM, vol. 113, no. 2, pp. 557-567, 2019, doi: 10.1007/s13398-018-0494-z.

[19] F. Qi, D.-W. Niu, and D. Lim, "Notes on the rodrigues formulas for two kinds of the Chebyshev polynomials," HAL archives, p. 6 pages, 2018. [Online]. Available: https://hal.archives-ouvertes.fr/hal-01705040

[20] F. Qi, D.-W. Niu, and Y.-H. Yao, "Special values of the Bell polynomials of the second kind for some sequences and functions," HAL archives, p. 25 pages, 2018. [Online]. Available: https://hal.archives-ouvertes.fr/hal-01766566

[21] F. Qi, X.-T. Shi, and B.-N. Guo, "Two explicit formulas of the Schröder numbers," Integers, vol. 16, pp. Paper No. A23, 15 pages, 2016. 
[22] F. Qi, X.-T. Shi, and F.-F. Liu, "Several identities involving the falling and rising factorials and the Cauchy, Lah, and Stirling numbers," Acta Univ. Sapientiae Math., vol. 8, no. 2, pp. 282-297, 2016, doi: 10.1515/ausm-2016-0019.

[23] F. Qi, X.-T. Shi, F.-F. Liu, and D. V. Kruchinin, "Several formulas for special values of the Bell polynomials of the second kind and applications," J. Appl. Anal. Comput., vol. 7, no. 3, pp. 857871, 2017, doi: 10.11948/2017054.

[24] F. Qi and V. Čerňanová, "Some discussions on a kind of improper integrals," Int. J. Anal. Appl., vol. 11, no. 2, pp. 101-109, 2016.

[25] F. Qi, V. Čerňanová, X.-T. Shi, and B.-N. Guo, "Some properties of central Delannoy numbers," J. Comput. Appl. Math., vol. 328, pp. 101-115, 2018, doi: 10.1016/j.cam.2017.07.013.

[26] F. Qi and Y.-H. Yao, "Simplifying coefficients in differential equations for generating function of Catalan numbers," Journal of Taibah University for Science, vol. 13, no. 1, pp. 947-950, 2019, doi: 10.1080/16583655.2019.1663782.

[27] F. Qi and M.-M. Zheng, "Explicit expressions for a family of the Bell polynomials and applications," Appl. Math. Comput., vol. 258, pp. 597-607, 2015, doi: 10.1016/j.amc.2015.02.027.

[28] F. Qi, Q. Zou, and B.-N. Guo, "The inverse of a triangular matrix and several identities of the Catalan numbers," Appl. Anal. Discrete Math., vol. 14, no. 1, p. in press, 2020, doi: 10.20944/preprints201703.0209.v2.

[29] C.-F. Wei and F. Qi, "Several closed expressions for the Euler numbers," J. Inequal. Appl., pp. 2015:219, 8 pages, 2015, doi: 10.1186/s13660-015-0738-9.

[30] J.-L. Zhao and F. Qi, "Two explicit formulas for the generalized Motzkin numbers," J. Inequal. Appl., pp. Paper No. 44, 8 pages, 2017, doi: 10.1186/s13660-017-1313-3.

[31] D. Zwillinger, CRC standard mathematical tables and formulae, 2nd ed. CRC Press, Boca Raton, FL, 2012.

Authors' addresses

\section{Feng Qi}

Institute of Mathematics, Henan Polytechnic University, Jiaozuo 454010, Henan, China; College of Mathematics, Inner Mongolia University for Nationalities, Tongliao 028043, Inner Mongolia, China; School of Mathematical Sciences, Tianjin Polytechnic University, Tianjin 300387, China

E-mail address: qifeng618@gmail.com

URL: https: //qifeng618.wordpress.com

\section{Da-Wei Niu}

Department of Science, Henan University of Animal Husbandry and Economy, Zhengzhou 450046, Henan, China

E-mail address: nnddwwegmail.com

URL: https://orcid.org/0000-0003-4033-7911

Dongkyu Lim

Department of Mathematics Education, Andong National University, Andong 36729, Republic of Korea

E-mail address: dgrim84@gmail.com

URL: https://orcid.org/0000-0002-0928-8480 